PROCEEDINGS OF THE

AMERICAN MATHEMATICAL SOCIETY

Volume 129, Number 5, Pages 1551-1562

S 0002-9939(00)05661-6

Article electronically published on October 24, 2000

\title{
A NEW CONSTRUCTION OF SEMI-FREE ACTIONS ON MENGER MANIFOLDS
}

\author{
SERGEI M. AGEEV AND DUŠAN REPOVŠ
}

(Communicated by Alan Dow)

\begin{abstract}
A new construction of semi-free actions on Menger manifolds is presented. As an application we prove a theorem about simultaneous coexistence of countably many semi-free actions of compact metric zero-dimensional groups with the prescribed fixed-point sets: Let $G$ be a compact metric zerodimensional group, represented as the direct product of subgroups $G_{i}, M$ a $\mu^{n}$ manifold and $\nu(M)$ (resp., $\Sigma(M))$ its pseudo-interior (resp., pseudo-boundary). Then, given closed subsets $X_{i}, i \geq 1$, of $M$, there exists a $G$-action on $M$ such that (1) $\nu(M)$ and $\Sigma(M)$ are invariant subsets of $M$; and (2) each $X_{i}$ is the fixed point set of any element $g \in G_{i} \backslash\{e\}$.
\end{abstract}

\section{InTRODUCTION}

The following remarkable theorem was proved by Iwamoto [11]:

Theorem (0.1). Let $G$ be a compact metric zero-dimensional group, $M$ a $\mu^{n}$ manifold and $\nu(M)$ (resp. $\Sigma(M)$ ) its pseudo-interior (resp., pseudo-boundary). Then for every closed subset $X$ of $M$, there exists a semi-free $G$-action on $M$ such that $X$ is the fixed-point set of every element $g \in G \backslash\{e\}$, and $\nu(M)$ and $\Sigma(M)$ are invariant subsets of $M$.

This theorem is a significant generalization of the Dranishnikov free action theorem 8 and Sakai's results [13, 14. In the present paper we give a new construction of semi-free actions, and prove a simultaneous coexistence of countably many semi-free actions of compact metric zero-dimensional groups with the prescribed fixed-point sets.

Theorem (0.2). Let $G$ be a compact metric zero-dimensional group, represented as the direct product $\prod G_{i}$ of subgroups $G_{i}, M a \mu^{n}$-manifold and $\nu(M)$ (resp., $\Sigma(M)$ ) its pseudo-interior (resp., pseudo-boundary). Then, given closed subsets $X_{i}, i \geq 1$, of $M$, there exists a $G$-action on $M$ such that:

(1) $\nu(M)$ and $\Sigma(M)$ are invariant subsets of $M$; and

(2) each $X_{i}$ is the fixed-point set of every element $g \in G_{i} \backslash\{e\} \subset \prod G_{i}$.

Recall that an action of $G$ on a space $X$ is a homomorphism $T: G \rightarrow$ Aut $X$ of the group $G$ into the group Aut $X$ of all autohomeomorphisms of $X$, such that the

Received by the editors May 22, 1998 and, in revised form, August 12, 1999.

1991 Mathematics Subject Classification. Primary 57S10, 54C55.

Key words and phrases. Semi-free action, Menger manifold, absolute extensor in finite dimension. 
map $G \times X \rightarrow X$, given by $(g, x) \mapsto T(g)(x)=g \cdot x$, is continuous. A space $X$ with a fixed action of $G$ is called a $G$-space.

A subset $A \subset X$ (resp., a point $a \in X$ ) is said to be invariant (resp., fixed) if $G \cdot A=\{g \cdot a \mid g \in G, a \in A\}=A$ (resp., $G \cdot a=a$ ). A $G$-space $X$ is said to be free (resp., semi-free) if $g \cdot x \neq x$, for every $x \in X$ and $g \neq e$ (resp., $g \cdot x \neq x$, for every nonfixed point $x$ and $g \neq e$ ).

The proof of Theorem (0.2) is presented in detail only for the case of the Menger compactum $\mu^{n}$. By Pontryagin's theorem [12, each $G_{i}$ can be considered as a closed subgroup of the product $\prod_{k=1}^{\infty} H_{i k}$ of nontrivial finite groups with the following property: Every nontrivial element $g_{i}=\left(g_{i k}\right) \in G_{i}$ has infinitely many nontrivial coordinates.

Next, fix the Cantor compactum $\mathcal{C}=\mu^{0}$ with the pseudo-interior $\nu=\nu\left(\mu^{0}\right)$ and the pseudo-boundary $\Sigma=\Sigma\left(\mu^{0}\right)$. The key to the construction of the desired semi-free action lies in the canonical surjection

$$
r: I^{n} \times H \times \mathcal{C} \rightarrow \mu^{n}, \text { where } H=\prod_{i} H_{i} \text { and } H_{i}=\prod_{k} H_{i k},
$$

and a discontinuous action $\Psi$ of $H$ on $I^{n} \times H \times \mathcal{C}$ such that:

(3) the restriction of $\Psi$ on $G_{k} \subset H_{k}$ is a discontinuous semi-free action with respect to $r^{-1}\left(X_{k}\right)$;

(4) $\Psi(h,(x, u, v))=\left(x, u^{\prime}, v\right)$, where $h \in H, x \in I^{n}, u \in H, v \in \mathcal{C}$ (i.e. $\Psi$ changes only the middle coordinate);

(5) $r\left(I^{n} \times H \times \nu\right)$ is the pseudo-interior $\nu\left(\mu^{n}\right)$ and $r\left(I^{n} \times H \times \Sigma\right)$ is the pseudoboundary $\Sigma\left(\mu^{n}\right)$;

(6) $r^{-1}(m)$ is a rectangle subset of $I^{n} \times H \times \mathcal{C}$, for every $m \in \mu^{n}$; and

(7) $\Phi(h, r(x, u, v))=r\left(x, u^{\prime}, v\right)$, where $\left(x, u^{\prime}, v\right)=\Psi(h,(x, u, v))$, is a continuous action of $H$ on $\mu^{n}$, and the restriction of $\Phi$ on $G_{k}$ is a continuous semi-free action with respect to $X_{k}$.

The proof of Theorem (0.2) in the general case (for an arbitrary $\mu^{n}$-manifold) is analogous to the special case of $\mu^{n}$ and will be discussed in the last chapter.

\section{Preliminaries}

All spaces are assumed to be separable metric and all maps to be continuous. Recall that a space $X$ is said to be $(k-1)$-connected $\left(\mathrm{C}^{k-1}\right)$ if the homotopy group $\pi_{i}(X)$ is trivial, for every $i<k$. A space $X$ is said to be locally $(k-1)$ connected $\left(\mathrm{LC}^{k-1}\right)$ if for every $x \in X$ and every neighborhood $U \ni x$, there exists a neighborhood $V \ni x$ with the property that every map $\alpha: S^{i}=\partial B^{i+1} \rightarrow V, i<k$, extends to $\tilde{\alpha}: B^{i+1} \rightarrow U$.

By the Kuratowski-Dugundji theorem [5], $X \in \mathrm{LC}^{k-1} \cap \mathrm{C}^{k-1}$ (resp., $X \in \mathrm{LC}^{k-1}$ ) if and only if $X \in \operatorname{AE}(k)$, i.e. $X$ is an absolute extensor in dimension $k$ (resp., $X \in \operatorname{ANE}(k)$, i.e. $X$ is an absolute neighborhood extensor in dimension $k$ ). A family $\left\{X_{\alpha}\right\}$ of sets $X_{\alpha} \subset X$ is said to be equi-LC ${ }^{k-1}$ if for every $x \in \bigcup X_{\alpha}$ and every neighborhood $U \ni x$, there exists a neighborhood $V \ni x$ with the property that every partial map $Z \hookleftarrow A \stackrel{f}{\rightarrow} V \cap X_{\alpha}, \operatorname{dim} Z \leq k$, extends to $g: Z \rightarrow U \cap X_{\alpha},\left.g\right|_{A}=f$. The following criteria are convenient for verification of connectivity properties of spaces (cf. [1] and [4], respectively): 
Proposition (1.1). Let $\left\{Z_{\alpha}\right\}$ be a closed cover of the compactum $Z$. Then the following assertions hold:

(a) $Z \in \mathrm{AE}(n)$ if and only if for every neighborhood $U(z)$ of $z \in Z$, there exists a neighborhood $V(z)$ (if $U(z)=Z$ then we require $V(z)=Z$ ), such that for every $\operatorname{map} \varphi: S^{k} \rightarrow V(z), k<n$, and for every $\nu>0$, there exists a map $\psi: B^{k+1} \rightarrow Z$, with $\left(\varphi,\left.\psi\right|_{S^{k}}\right)<\nu$.

(b) $\left\{Z_{\alpha}\right\} \in$ equi-LC ${ }^{n-1}$, if and only if for every neighborhood $U(z)$ of $z \in Z$, there exists a neighborhood $V(z)$, such that for every map $\varphi: S^{k} \rightarrow V(z) \cap Z_{\alpha}, k<n$, and for every $\nu>0$, there exists a map $\psi: B^{k+1} \rightarrow U(z) \cap Z_{\alpha}$ with $\left(\varphi,\left.\psi\right|_{S^{k}}\right)<\nu$.

Proposition (1.2). Let $\mathcal{P}=\left\{p_{\alpha}\right\}$ be a closed cover of an $\operatorname{ANE}(r)$-compactum $Z$ and suppose that $p_{\alpha_{1}} \cap p_{\alpha_{2}} \cap \cdots \cap p_{\alpha_{t}} \neq \emptyset$ implies $p_{\alpha_{1}} \cap p_{\alpha_{2}} \cap \cdots \cap p_{\alpha_{t}} \in \operatorname{AE}(r+1-t)$, for every $t \leq r$. Then $Z \in \mathrm{AE}(r)$ if and only if the nerve $\mathcal{N}\langle\mathcal{P}\rangle$ of $\mathcal{P}$ is $(r-1)$ connected.

We shall need the following three results of Bestvina [3].

Theorem (1.3). A locally compact space $X$ is $\mu^{k}$-manifold if and only if $X \in$ $\operatorname{ANE}(k), \operatorname{dim} X=k$ and $X$ has the disjoint $k$-disks property, namely $X \in \mathrm{DD}^{k} \mathrm{P}$. If, in addition, $X$ is a compact $\mathrm{AE}(k)$, then $X$ is homeomorphic to the Menger universal compactum $\mu^{k}$.

Theorem (1.4). For every $\mu^{k}$-manifold $M^{k}$, there exists a PL manifold $R$ of dimension $\geq 2 k+1$ with a triangulation $L$ and a proper map $f: R^{(k)} \rightarrow M$ which induces isomorphisms of homotopy groups of $\operatorname{dim}<k$ and homotopy groups of ends of $\operatorname{dim}<k$ (here $R^{(k)}$ is the $k$-skeleton with respect to $L$ ).

Theorem (1.5). Let $f: M_{1} \rightarrow M_{2}$ be a proper map between $\mu^{k}$-manifolds which induces isomorphisms of homotopy groups of $\operatorname{dim}<k$ and homotopy groups of ends of $\operatorname{dim}<k$. Then $f$ is properly $(k-1)$-homotopic to a homeomorphism.

Let $M$ be a $\mu^{k}$-manifold. By $\mathcal{Z}_{M}$, we shall denote the collection of all $Z$-sets

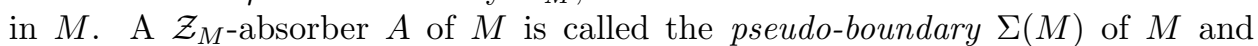
the complement $M \backslash A$ is called the pseudo-interior $\nu(M)$ of $M$. The topological types of the pseudo-boundaries and the pseudo-interiors of $M$ are unique [2]. The following criterion was proved in [7].

Proposition (1.6). Let $\left\{A_{i}\right\}_{i=1}^{\infty}$ be an increasing sequence of $Z$-sets in a $\mu^{k}$ manifold $M$ with the following properties:

(1) for every $\epsilon>0$, there exists $m>0$ such that $A_{m}$ is $\epsilon$-dense in $M$;

(2) each $A_{i}$ is a $Z$-set in $A_{i+1}$;

(3) $\left\{A_{i}\right\}_{i=1}^{\infty}$ is equi-LC ${ }^{k-1}$ and

(4) $A_{i}$ is a $\mu^{k}$-manifold.

Then $\bigcup_{i=1}^{\infty} A_{i}$ is a pseudo-boundary of $M$.

The pseudo-boundary and the pseudo-interior of the zero-dimensional Menger manifold $\mathcal{C}=\mu^{0}$ can be described in the following manner:

Definition (1.7). (5) A subset $R \subset \prod_{i=1}^{s} T_{i}, 1 \leq s \leq \infty$, is called a rectangle, if $R=\prod_{i=1}^{s} T_{i}^{\prime}$, where $T_{i}^{\prime} \subset T_{i}$ for every $i$. 
(6) A rectangle subset $R_{1}=\prod_{i=1}^{s} T_{i}^{\prime}$ is said to have an infinite codimension in a rectangle subset $R_{2}=\prod_{i=1}^{s} T_{i}^{\prime \prime}$, whenever $R_{1} \subset R_{2}$ and the set $\{i \in \mathbb{N}$ | $\left.T_{i}^{\prime \prime} \backslash T_{i}^{\prime} \neq \emptyset\right\}$ is infinite.

Proposition (1.8). Let $\mathcal{C}=\prod_{i=1}^{\infty} T_{i}$ be the Cantor compactum. Then there exists a sequence of rectangle subsets $R_{1} \subset R_{2} \subset \ldots$ such that

(7) $R_{i} \cong \mathcal{C}$ for every $i$;

(8) every $R_{i}$ has infinite codimension in $R_{i+1}$; and

(9) $R=\bigcup_{i=1}^{\infty} R_{i}$ is a dense subset in $\mathcal{C}$.

According to Proposition (1.6), $R$ is the pseudo-boundary of $\mathcal{C}$.

\section{The Canonical Decomposition of $I^{n} \times \prod T_{i}$}

Let us consider a partition $\Delta_{m}, m \geq 1$, of the unit segment $I$, determined by the subset $\delta_{m}=\left\{\frac{2 i-1}{2^{m}} \mid 0<i \leq 2^{m-1}\right\}$. The product $\underbrace{\Delta_{m} \times \cdots \times \Delta_{m}}_{n}=\left(\Delta_{m}\right)^{n}$ of the partitions $\Delta_{m}$ is a partition of the cube $I^{n}=\underbrace{I \times \cdots \times I}_{n}$ into $\left(2^{m-1}+1\right)^{n}$ cubes of diameter $\leq \sqrt{n} \cdot 2^{1-m}$. The union $F_{m}$ of all boundaries of these cubes satisfies the following properties:

(1) $F_{m}=\left\{x=\left(x_{1}, \ldots, x_{n}\right) \in I^{n} \mid x_{i} \in \delta_{m}\right.$, for some $\left.i\right\}$; and

(2) for every $x \in I^{n}$, the set $\left\{m \mid x \in F_{m}\right\}$ has less than $n+1$ elements.

Fix a sequence $\left\{T_{i}, i \geq 1\right\}$ of finite sets $T_{i}$ with $\left|T_{i}\right|>1$, and construct (in a canonical way) an upper semi-continuous decomposition $\mathcal{T}$ of $Q=I^{n} \times \prod_{i=1}^{\infty} T_{i}$. The quotient space $P=Q / \mathcal{T}$, generated by $\mathcal{T}$, will have properties (3)-(5) below:

(3) $P$ is compact and $\operatorname{dim} P=n$;

(4) $P$ satisfies the disjoint $n$-disks property $\left(D D^{n} P\right)$; and

(5) $P \in \mathrm{C}^{n-1} \cap \mathrm{LC}^{n-1}$.

By Theorem (1.3), $P$ and the Menger compactum $\mu^{n}$ are homeomorphic. Let $x \in I^{n}$ and $t=\left(t_{i}\right)_{i=1}^{m} \in \prod_{i=1}^{m} T_{i}, 1 \leq m \leq \infty$. (Convention: $m+1=\infty$ in case $m=\infty$.) Let us denote $\stackrel{i=1}{\mathcal{T}_{i}}(x, t)=t_{i}$, if $x \notin F_{i} ; \mathcal{T}_{i}(x, t)=T_{i}$, if $x \in F_{i}$, and $\mathcal{T}(x, t)=\prod_{i=1}^{m} \mathcal{T}_{i}(x, t)$.

Proposition (2.1). The family $\mathcal{T}_{m}=\left\{x \times \mathcal{T}(x, t) \mid x \in I^{n}, t \in \prod_{i=1}^{m} T_{i}\right\}, 1 \leq m \leq \infty$, yields an upper semi-continuous decomposition of $Q_{m}=I^{n} \times \prod_{i=1}^{m} T_{i}$ :

(6) for every $\left(x_{0}, t_{0}\right)$ and $\epsilon>0$, there exists $\delta>0$ such that $\operatorname{dist}\left((x, t),\left(x_{0}, t_{0}\right)\right)<$ $\delta$ implies $\alpha_{H}\left(x \times \mathcal{T}(x, t), x_{0} \times \mathcal{T}\left(x_{0}, t_{0}\right)\right)<\epsilon\left(\right.$ here $\alpha_{H}(A, B)=\inf \{\gamma>0 \mid A \subset$ $\mathrm{N}(B ; \gamma)\}$ is the Hausdorff deflection; $c f$. [10, p. 98, (7.7.1)]). 
Proof. By definition, it is evident that

(7) $x \times \mathcal{T}(x, t) \cap x^{\prime} \times \mathcal{T}\left(x^{\prime}, t^{\prime}\right) \neq \emptyset \Longleftrightarrow x=x^{\prime}$ and $t_{i}=t_{i}^{\prime}$ for all $i<m+1$ such that $x \notin F_{i} \Longleftrightarrow x \times \mathcal{T}(x, t) \equiv x^{\prime} \times \mathcal{T}\left(x^{\prime}, t^{\prime}\right)$ (we use a convention that $m+1=\infty$ in case $m=\infty$ ).

Fix $x_{0} \in I^{n}, t_{0} \in \prod_{i=1}^{m} T_{i}$ and $\epsilon>0$. Let us choose $\delta>0$ and a finite number $p \leq m$ such that

(8) $\operatorname{dist}\left(x, x^{\prime}\right)<\delta$ and the coincidence of the first $p$ coordinates of $t$ and $t^{\prime}$ implies $\operatorname{dist}\left((x, t),\left(x^{\prime}, t^{\prime}\right)\right)<\epsilon / 2$

(9) $\operatorname{dist}\left((x, t),\left(x^{\prime}, t^{\prime}\right)\right)<\delta$ implies the coincidence of the first $p$ coordinates of $t$ and $t^{\prime}$ and, naturally, $\operatorname{dist}\left(x, x^{\prime}\right)<\delta$; and

(10) $\delta<\min \left\{\operatorname{dist}\left(x_{0}, F_{i}\right) \mid x_{0} \notin F_{i}, i \leq p\right\}$.

Let $\operatorname{dist}\left((x, t),\left(x_{0}, t_{0}\right)\right)<\delta$. It easily follows from (9) and (10) that $\operatorname{dist}\left(x, x_{0}\right)<\delta$, the first $p$ coordinates of $t$ and $t_{0}$ coincide and $\mathcal{T}_{i}(x, t) \subset \mathcal{T}_{i}\left(x_{0}, t\right)$, for every $i \leq p$. Then $\alpha_{H}\left(x \times \mathcal{T}(x, t), x_{0} \times \mathcal{T}\left(x_{0}, t\right)\right)<\epsilon / 2$ by (8). The coincidence of the first $p$ coordinates of $t$ and $t_{0}$ implies $\alpha_{H}\left(x_{0} \times \mathcal{T}\left(x_{0}, t\right), x_{0} \times \mathcal{T}\left(x_{0}, t_{0}\right)\right)<\epsilon / 2$. Hence it follows that $\alpha_{H}\left(x \times \mathcal{T}(x, t), x_{0} \times \mathcal{T}\left(x_{0}, t_{0}\right)\right) \leq \alpha_{H}\left(x \times \mathcal{T}(x, t), x_{0} \times \mathcal{T}\left(x_{0}, t\right)\right)+$ $\alpha_{H}\left(x_{0} \times \mathcal{T}\left(x_{0}, t\right), x_{0} \times \mathcal{T}\left(x_{0}, t_{0}\right)\right)<\epsilon$.

Corollary (2.2). For every $1 \leq m \leq \infty$, the following assertions hold:

(a) the quotient space $P_{m}=Q_{m} / \mathcal{T}_{m}$ is a compactum; and

(b) if $m<\infty$, then the map $p_{m}: P_{m} \rightarrow P_{m-1}$, defined by setting

$$
p_{m}\left(r_{m}\left(x,\left(t_{1}, \ldots, t_{m}\right)\right)\right)=r_{m}\left(x,\left(t_{1}, \ldots, t_{m-1}\right)\right),
$$

is well-defined and continuous and the following diagram is commutative:

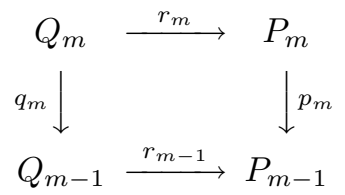

where $q_{m}$ is the projection on $Q_{m-1}=I^{n} \times \prod_{i=1}^{m-1} T_{i}$.

For $m=\infty$ we have the map $r: Q=I^{n} \times \prod_{i=1}^{\infty} T_{i} \rightarrow P=P_{\infty}$. Clearly, $P$ is the limit of the inverse system $\left\{P_{1} \stackrel{p_{2}}{\leftarrow} P_{2} \stackrel{p_{3}}{\leftarrow} P_{3} \stackrel{p_{4}}{\leftarrow} \ldots\right\}$. Since $r_{m}$ has finitely many fibers for $m<\infty$, it follows that $\operatorname{dim} P_{m}=\operatorname{dim} Q_{m}=n$. Since the dimension of the limit of the inverse system of $n$-dimensional spaces is less than or equal to $n$, we have $\operatorname{dim} P \leq n$.

Proposition (2.3). $\operatorname{dim} P=n$ and $P \in D D^{n} P$.

Proof. Every $a \in T_{m+1}$ naturally generates the embedding $s_{m, a}: P_{m} \rightarrow P_{m+1}$ of $P_{m}$ into $P_{m+1}$ defined by $s_{m, a}\left(r_{m}(x, t)\right)=r_{m+1}\left(x, t^{\prime}\right)$, where $t^{\prime}=(t, a)$. It is evident that $s_{m, a}$ is a section of $p_{m+1}$. Hence $P$ contains a copy of $P_{m}$ with $\operatorname{dim} P_{m}=n$. Therefore, $\operatorname{dim} P=n$.

One can easily conclude from (2) that the images of the following compositions are disjoint:

$$
\operatorname{Im}\left(s_{m+n, a_{m+n+1}} \circ \cdots \circ s_{m, a_{m+1}}\right) \cap \operatorname{Im}\left(s_{m+n, a_{m+n+1}^{\prime}} \circ \cdots \circ s_{m, a_{m+1}^{\prime}}\right)=\emptyset,
$$

for every $a_{m+n+1} \neq a_{m+n+1}^{\prime}, \ldots, a_{m+1} \neq a_{m+1}^{\prime}$. Consequently, $P$ satisfies $D D^{n} P$. 
Remark (2.4). If $R^{\prime}$ is a rectangle subset of infinite codimension in a rectangle subset $R^{\prime \prime}$ of $\prod T_{i}$, then $P^{\prime}=r\left(I^{n} \times R^{\prime}\right)$ is a $Z$-set in $P^{\prime \prime}=r\left(I^{n} \times R^{\prime \prime}\right)$ and $P^{\prime \prime} \in \mathrm{DD}^{n} \mathrm{P}$.

In the next section we shall prove that $P^{\prime \prime} \in \mathrm{AE}(n)$; so it will follow by the Bestvina theorem (1.3) that $P^{\prime \prime}$ is homeomorphic to the Menger compactum $\mu^{n}$.

\section{Connectivity properties of subsets of $P$}

Let the factor $T_{p}, p<m+1$, be represented as the disjoint union $T_{p}^{\prime} \amalg T_{p}^{\prime \prime}$ (we assume $m+1=m$ for $m=\infty$ ). By replacing $T_{p}$ with $T_{p}^{\prime}$, we obtain the decomposition $\mathcal{T}_{m}^{\prime}$ of $Q_{m}^{\prime}=I^{n} \times T_{p}^{\prime} \times \prod\left\{T_{i} \mid i<m+1, i \neq p\right\}, 1 \leq m \leq \infty$, and the quotient map $r_{m}^{\prime}: Q_{m}^{\prime} \rightarrow P_{m}^{\prime}=Q_{m}^{\prime} / \mathcal{T}_{m}^{\prime}$. The decomposition $\mathcal{T}_{m}^{\prime \prime}$ of the space $Q_{m}^{\prime \prime}$ and the map $r_{m}^{\prime \prime}: Q_{m}^{\prime \prime} \rightarrow P_{m}^{\prime \prime}$ are defined analogously. Note that $Q_{m}^{\prime} \amalg Q_{m}^{\prime \prime}=Q_{m}$. It is clear that $r_{m}\left(Q_{m}^{\prime}\right)=\hat{P}_{m}^{\prime} \subset P_{m}$ and $r_{m}\left(Q_{m}^{\prime \prime}\right)=\hat{P}_{m}^{\prime \prime} \subset P_{m}$ are naturally homeomorphic to $P_{m}^{\prime}$ and $P_{m}^{\prime \prime}$, respectively.

Proposition (3.1). $\hat{P}_{m}^{\prime} \cap \hat{P}_{m}^{\prime \prime}=r_{m}\left(F_{p} \times \prod_{i=1}^{m} T_{i}\right)$.

Definition (3.2). An index of $x \in I$ (briefly ind $x$ ) is defined to be $i \in \mathbb{N}$, if $x \in \delta_{i}$, and $\infty$, if $x \notin \delta_{j}$ for every $j$. An index of $b=\left(b_{1}, \ldots, b_{n}\right) \in I^{n}$ is defined to be $\operatorname{ind}(b)=\left\{\operatorname{ind}\left(b_{i}\right) \mid i \leq n\right\} \subset \mathbb{N} \cup\{\infty\}$.

Let $J$ be a closed segment from the partition $\Delta_{m}, m<\infty$. If $J \subset \operatorname{Int} I$, then there exists a unique point $b^{\prime} \in J$ with $\operatorname{ind}\left(b^{\prime}\right)<m\left(b^{\prime}\right.$ is the midpoint of $J$ ). If $J \cap\{0,1\}=b^{\prime}$, then $J$ does not contain points with index less than $m$. We define $\operatorname{Ind}(J)=\operatorname{ind}\left(b^{\prime}\right)$ in the first case and $\operatorname{Ind}(J)=\infty$ in the second one. In both cases point $b^{\prime}$ is said to be the center of $J$. Let $J=\left[\frac{2 k+1}{2^{m}}, \frac{2 k+3}{2^{m}}\right] \in \Delta_{m}$. Then $\operatorname{Ind}(J)<m$ and $b^{\prime}=\frac{2 k+2}{2^{m}}$ is the center of $J$.

The partition $\left(\Delta_{m}\right)^{n}$ of $I^{n}$ yields new partitions $\mathcal{Q}_{m}^{\prime}$ of $Q_{m}=I^{n} \times \prod_{i=1}^{m} T_{i}$ and $\mathcal{Q}_{m}$ of $Q=I^{n} \times \prod_{i=1}^{\infty} T_{i}$ defined as follows:

$$
\begin{gathered}
\mathcal{Q}_{m}^{\prime}=\left\{\mathcal{E}^{\prime}=\mathcal{J} \times \mathcal{T}(b, t) \mid \mathcal{J}=\prod_{i=1}^{n} J_{i} \in\left(\Delta_{m}\right)^{n}, b=\left(b_{i}\right) \in I^{n},\right. \\
\left.t \in \prod_{i=1}^{m} T_{i}, \text { each } b_{i} \text { is the center of } J_{i}\right\}
\end{gathered}
$$

and $\mathcal{Q}_{m}=\left\{\mathcal{E}^{\prime} \times \prod_{i=m+1}^{\infty} T_{i} \mid \mathcal{E}^{\prime} \in \mathcal{Q}_{m}^{\prime}\right\}$. The partitions $\mathcal{Q}_{m}$ of $Q$ and $\mathcal{Q}_{m}^{\prime}$ of $Q_{m}$ consist of regular closed subsets.

Theorem (3.3). Let $\mathcal{E}^{\prime}=\mathcal{J} \times \mathcal{T}(b, t)$ be an arbitrary element of the partition $\mathcal{Q}_{m}^{\prime}, m<\infty$, and $\mathcal{J}_{p_{1} \ldots p_{w}}=\mathcal{J} \cap F_{p_{1} \ldots p_{w}} \neq \emptyset$, where $1 \leq p_{1}<\cdots<p_{w} \leq s, s \geq$ $m, 0 \leq w \leq n$, and

$$
F_{p_{1} \ldots p_{w}}=\left\{\begin{array}{l}
\bigcap_{k=1}^{w} F_{p_{k}} \text { if } w \geq 1 \\
I^{n} \quad \text { if } w=0
\end{array}\right.
$$


Then for every rectangle subset $R=\prod_{i=1}^{s} T_{i}^{\prime}$ with the property $(*)$ :

$$
\text { " }\left|T_{i}^{\prime}\right|>1 \text { implies } i \geq m \text { or } i \in \operatorname{ind}(b) \text { ", }
$$

the compactum $X=r_{s}\left(\mathcal{J}_{p_{1} \ldots p_{w}} \times R\right)$ is a nonempty $\operatorname{AE}(n-w)$.

Corollary (3.4). Let $\mathcal{E}^{\prime}=\mathcal{J} \times \mathcal{T}(b, t) \in \mathcal{Q}_{m}^{\prime}, m<\infty$, and $m \leq p_{1}<\cdots<p_{w} \leq$ $s, 0 \leq w \leq n$. Then for rectangle subsets $R_{1} \subset \prod_{i=1}^{m} T_{i}$ and $R_{2} \subset \prod_{i=m+1}^{s} T_{i}$, the compactum $r_{s}\left(\mathcal{J}_{p_{1} \ldots p_{w}} \times\left(\mathcal{T}(b, t) \cap R_{1}\right) \times R_{2}\right)$ is a nonempty $\operatorname{AE}(n-w)$.

The following preliminary facts precede the proof of Theorem (3.3). Let us consider a finite set $A$ represented as the disjoint union $A_{1} \amalg \cdots \amalg A_{w}$. We shall study the connectivity properties of the subpolyhedron $K=K_{A_{1} \ldots A_{w}}^{v}, v \leq w$, of the $(|A|-1)$-dimensional simplex $\Delta: \quad K=\bigcup\left\{\left\langle a_{1} \ldots a_{v}\right\rangle \mid\left\langle a_{1} \ldots a_{v}\right\rangle\right.$ is a $(v-1)$ dimensional simplex spanned by the vertices $a_{k} \in A_{i_{k}}, 1 \leq k \leq v$, where $i_{k} \neq i_{k^{\prime}}$ if $\left.k \neq k^{\prime}\right\}$.

Lemma (3.5). $K_{A_{1} \ldots A_{w}}^{v} \in C^{v-2}$ for each $v \leq w$.

Proof. We now proceed by induction on $\theta=e \cdot w+f+v$, where $e=\max \left|A_{i}\right|, f=$ $\left|\left\{i:\left|A_{i}\right|=e\right\}\right|$. It is evident that $\theta^{\prime}=e^{\prime} \cdot w^{\prime}+f^{\prime}+v^{\prime}<\theta$ if $e^{\prime}<e$ and $w^{\prime} \leq w, v^{\prime} \leq v$ or $e^{\prime} \leq e, f^{\prime}<f$ and $w^{\prime} \leq w, v^{\prime} \leq v$.

The basis of the induction corresponds to $\theta=1 \cdot v+v+v=3 v$. In this case $K$ is the $(v-1)$-skeleton of $\Delta$, which is $(v-2)$-connected. The same is valid for $e=1$.

Assume the validity of the lemma for all $\theta^{\prime}<\theta$. Suppose, without loss of generality, that $\left|A_{1}\right|=e>1$ and $A_{1}=A_{1}^{\prime} \amalg A_{1}^{\prime \prime},\left|A_{1}^{\prime}\right| \leq\left|A_{1}^{\prime \prime}\right|<e$. By the inductive hypothesis $\left(\theta^{\prime}<\theta\right.$ and $\left.\theta^{\prime \prime}<\theta\right), K_{A_{1}^{\prime} A_{2} \ldots A_{w}}^{v}$ and $K_{A_{1}^{\prime \prime} A_{2} \ldots A_{w}}^{v}$ are $(v-2)$-connected and their intersection

$$
K_{A_{1}^{\prime} A_{2} \ldots A_{w}}^{v} \cap K_{A_{1}^{\prime \prime} A_{2} \ldots A_{w}}^{v}=K_{A_{2} \ldots A_{w}}^{v} \cup K_{A_{2} \ldots A_{w}}^{v-1}= \begin{cases}K_{A_{2} \ldots A_{w}}^{v} \text { if } w \geq v ; \\ K_{A_{2} \ldots A_{w}}^{v-1} \text { if } w=v \geq 2 .\end{cases}
$$

By the Van Kampen and the Mayer-Vietoris argument [15], $K_{A_{1} A_{2} \ldots A_{w}}^{v} \in \mathrm{C}^{v-2}$.

Lemma (3.6). Let $\mathcal{J}=\prod_{i=1}^{n} J_{i} \in\left(\Delta_{m}\right)^{n}, b=\left(b_{i}\right) \in I^{n}$, each $b_{i}$ is the center of $J_{i}$, and $\mathcal{J}_{p_{1} \ldots p_{w}} \neq \emptyset$ for $1 \leq p_{1}<\cdots<p_{w}, 0 \leq w \leq n$. Then $\mathcal{J}_{p_{1} \ldots p_{w}} \in \operatorname{AE}(n-w)$.

Proof. Let $\pi_{i}: I^{n} \rightarrow I$ be the projection onto the $i$ th factor. Then

$$
F_{p_{1}}=\bigcup_{k=1}^{n} \coprod_{q \in \delta_{p_{1}}} \Pi_{k, q} \text {, where } \Pi_{k, q}=\pi_{k}^{-1}(q)=\left\{\left(x_{1}, \ldots, x_{n}\right) \in I^{n} \mid x_{k}=q\right\} .
$$

Hence $\mathcal{J}_{p_{1} \ldots p_{w}}=\bigcup_{k=1}^{n} \amalg\left\{\Pi_{k, q}^{\prime} \mid q \in A_{k}\right\}$, where $\Pi_{k, q}^{\prime}=\mathcal{J} \cap \Pi_{k, q} \cap F_{p_{2} \ldots p_{w}}, A_{k}=\{q \in$ $\left.\delta_{p_{1}} \mid \Pi_{k, q}^{\prime} \neq \emptyset\right\}$. It is easy to see that $\mathcal{J}_{p_{1} \ldots p_{w}} \neq \emptyset$ implies $p_{k} \geq m$ or $p_{k} \in \operatorname{ind}(b)$, for every $k \leq w$.

We shall omit the proof of the following fact which easily follows from the note mentioned above:

Lemma (3.7). $\bigcap_{i=1}^{r} \Pi_{k_{i} q_{i}}^{\prime} \neq \emptyset$ for every $k_{1}<k_{2}<\cdots<k_{r}, 0 \leq r \leq v=n+1-$ $w, q_{i} \in A_{k_{i}}$. 
We now proceed by induction on $\sigma=n+w$. The basis of induction is obvious. Assume the validity of the lemma for all $\sigma^{\prime}<\sigma$. Then $\Pi_{k, q}^{\prime} \in \operatorname{AE}((n-1)-(w-1))$ and moreover, for every $k_{1}<k_{2}<\cdots<k_{r}, 0 \leq r \leq v=n+1-w, q_{i} \in A_{k_{i}}$, we have $\bigcap_{i=1}^{r} \Pi_{k_{i} q_{i}}^{\prime}=\left(\mathcal{J} \cap \bigcap_{i=1}^{r} \Pi_{k_{i} q_{i}}\right) \cap F_{p_{2}} \cap \cdots \cap F_{p_{w}} \in \operatorname{AE}((n-r)-(w-1))=\operatorname{AE}((n-w+1)-r)$ (since $\bigcap_{i=1}^{r} \Pi_{k_{i} q_{i}}$ is an $(n-r)$-dimensional cube, parallel the $(n-r)$-dimensional plane $\left\{\left(x_{1}, \ldots, x_{n}\right) \mid x_{k_{i}}=0, i \leq r\right\}$ and $\mathcal{J} \cap \bigcap_{i=1}^{r} \Pi_{k_{i} q_{i}}$ is a nonempty element of $\left.\left(\Delta_{m}\right)^{n-r}\right)$.

Hence the intersections of the $\Pi_{k_{i} q_{i}}$ 's are correctly connected (in the sense of Bestvina [3]) and their nerve coincides with $K_{A_{1} \ldots A_{n}}^{v}=K_{A_{1} \ldots A_{n}}^{n+1-w}$, which is $\operatorname{AE}(v-1)=\operatorname{AE}(n-w)$ by Lemma (3.5).

To complete the proof we apply Proposition (1.2) to the cover $\left\{\Pi_{k q}^{\prime}\right\}$ of $F_{p_{1} \ldots p_{w}}$.

Proof of Theorem (3.3). We prove by induction on $\theta=s \cdot e+f-w$, where $e=$ $\max \left\{\left|T_{i}^{\prime}\right|: 1 \leq i \leq s\right\}, f=\left|\left\{i:\left|T_{i}^{\prime}\right|=e, 1 \leq i \leq s,\right\}\right|$ (e,f,s and $w$ are variables). Let us note that $\theta^{\prime}=s^{\prime} \cdot e^{\prime}+f^{\prime}-w^{\prime}<\theta$ provided $e^{\prime}=1, s^{\prime}=m, f^{\prime}=m$ and $w^{\prime}=n$ or $e^{\prime}=e, s^{\prime}=s, f^{\prime}<f$ and $w^{\prime}=w$ or $e^{\prime}<e, s^{\prime}=s$ and $w^{\prime}=w$ or $e^{\prime}=e, s^{\prime}=s, f^{\prime}=f$ and $w^{\prime}=w$.

If $e=1$, then each $\left|T_{i}\right|=1, i>m$, and therefore $X_{p_{1} \ldots p_{w}} \cong \mathcal{J}_{p_{1} \ldots p_{w}}$ which is $\mathrm{AE}(n-w)$ by Lemma (3.6). Hence the basis of the induction is verified.

Assume the validity of Theorem (3.3) for all $\theta^{\prime}<\theta$ and let $e>1$. Pick $i_{0} \leq s$ with $\left|T_{i_{0}}^{\prime}\right|=e$ and represent $T_{i_{0}}^{\prime}=\widehat{T}_{i_{0}} \amalg \check{T}_{i_{0}},\left|\widehat{T}_{i_{0}}\right| \leq\left|\check{T}_{i_{0}}\right|<e$. Then $X_{p_{1} \ldots p_{w}}$ is the union of

$$
\begin{gathered}
Y^{\prime}=X_{p_{1} \ldots p_{w}}^{\prime}=r_{s}\left(\mathcal{J}_{p_{1} \ldots p_{w}} \times \widehat{R}\right) \subset \widehat{P}_{s}^{\prime} \text { and } \\
Y^{\prime \prime}=X_{p_{1} \ldots p_{w}}^{\prime \prime}=r_{s}\left(\mathcal{J}_{p_{1} \ldots p_{w}} \times \check{R}\right) \subset \widehat{P}_{s}^{\prime \prime},
\end{gathered}
$$

where the rectangle subsets $\widehat{R}$ and $\check{R}$ coincide with the rectangle subsets $R \cap$ $\left(\prod_{i \neq i_{0}} T_{i} \times \widehat{T}_{i_{0}}\right)$ and $R \cap\left(\prod_{i \neq i_{0}} T_{i} \times \check{T}_{i_{0}}\right)$, which satisfy the property (*) (for notation see the beginning of Section 3). By the inductive hypothesis $\left(\theta^{\prime} \leq \theta^{\prime \prime}<\theta\right)$, we have $Y^{\prime}, Y^{\prime \prime} \in \operatorname{AE}(n-w)$. Let us prove that $Y^{\prime} \cap Y^{\prime \prime} \in \operatorname{AE}(n-w-1)$. It will then follow by the Van Kampen and the Mayer-Vietoris argument [15], that $X_{p_{1} \ldots p_{w}}=Y^{\prime} \cup Y^{\prime \prime} \in \mathrm{AE}(n-w)$.

The proof splits naturally into two parts: $i_{0} \in\left\{p_{1} \ldots p_{w}\right\}$ and $i_{0} \notin\left\{p_{1} \ldots p_{w}\right\}$.

Part (1). $i_{0} \notin\left\{p_{1} \ldots p_{w}\right\}$. In this case, $Y^{\prime} \cap Y^{\prime \prime} \cong r_{s}\left(\mathcal{J}_{p_{1} \ldots p_{w} i_{0}} \times R\right)$. Since $w^{\prime}=w+1$ and $e^{\prime}=e, s^{\prime}=s, f^{\prime}=f$, the inductive variable $\theta^{\prime}$ for $Y^{\prime} \cap Y^{\prime \prime}$ is less than $\theta$. By the inductive assumption $Y^{\prime} \cap Y^{\prime \prime} \in \mathrm{AE}(n-w-1)$.

Part (2). $i_{0} \in\left\{p_{1} \ldots p_{w}\right\}$. This implies that $Y^{\prime}=r_{s}\left(\mathcal{J}_{p_{1} \ldots p_{w}} \times \widehat{R}\right) \cong$ $r_{s}\left(\mathcal{J}_{p_{1} \ldots p_{w}} \times R\right)=X_{p_{1} \ldots p_{w}}$. Hence $X_{p_{1} \ldots p_{w}} \in \operatorname{AE}(n-w)$.

Theorem (3.8). Let $\mathcal{R}$ be the family of all rectangle subsets $R \subset \prod_{i=1}^{\infty} T_{i}$. Then

(a) $P_{R}=r\left(I^{n} \times R\right)$ is an $\mathrm{AE}(n)$, for every $R \in \mathcal{R}$; and

(b) $\left\{P_{R}=r\left(I^{n} \times R\right) \mid R \in \mathcal{R}\right\}$ is an equi-LC ${ }^{n-1}$-family.

Proof. (a) Fix a point $(x, t) \in I^{n} \times R$ and $r(x, t)=z$. For every $\epsilon>0$, it is possible to choose a number $m \geq 1$ and an element $\mathcal{E}^{\prime}=\mathcal{J} \times \mathcal{T}(b, t) \in \mathcal{Q}_{m}^{\prime}$ such that 
(1) the neighborhood $U=\mathrm{N}(z, \epsilon) \cap P_{R}$ of $z$ in $P_{R}$ contains a closed neighborhood $V=r\left(\mathcal{J} \times\left(\mathcal{T}(b, t) \cap R_{1}\right) \times R_{2} \times R_{3}\right)$ of $z$ in $P_{R}$, where $R$ is represented as the product $R_{1} \times R_{2} \times R_{3}, R_{1}=\prod_{i=1}^{m} T_{i}^{\prime}, R_{2}=\prod_{i=m+1}^{s} T_{i}^{\prime}$ and $R_{3}=\prod_{i=s+1}^{\infty} T_{i}^{\prime}(s$ is a sufficiently large number).

By Corollary (3.4), $V_{s}=r_{s}\left(\mathcal{J} \times\left(\mathcal{T}(b, t) \cap R_{1}\right) \times R_{2}\right) \subset P_{s}$ is $\operatorname{AE}(n)$ for every $s$. Pick an arbitrary constant $\nu>0$ and $s$ such that $\operatorname{Id}_{P}$ and the projection $p_{s}^{\infty}$ are $\nu$-close. Since $P_{s}$ is naturally embedded into $P$ (cf. Proposition (2.3)), we can consider $V_{s}$ as a subset of $V$. Now let $\varphi: S^{k} \rightarrow V, k<n$, be an arbitrary map. The composition $p_{s}^{\infty} \circ \varphi: S^{k} \rightarrow V_{s}$, which is $\nu$-close to $\varphi$, is extended on $\psi: B^{k+1} \rightarrow V_{s} \hookrightarrow V$. By Proposition (1.1a), $P_{R} \in \mathrm{AE}(n)$.

(b) The proof is analogous to (a), because the choice of the constant $\nu>0$ does not depend on the choice of the family $\left\{P_{R}\right\}$.

Corollary (3.9). $P=r(Q) \in \operatorname{AE}(n)$ (and hence, by Section 2, is also homeomorphic to $\left.\mu^{n}\right)$.

\section{Construction of the action of the zero-dimensional COMPACT GROUP $G$ ON $P$}

By Pontryagin's theorem [12, every compact metric zero-dimensional group $G_{i}$ can be considered as a closed subgroup of the product $\prod_{k=1}^{\infty} H_{i k}$ of nontrivial finite groups $H_{i k}$ with the following property:

(1) every nontrivial element $g_{i}=\left(g_{i k}\right) \in G_{i}$ has infinitely many nontrivial coordinates.

Let us identify some factors of $Q=I^{n} \times \prod_{i=1}^{\infty} T_{i}$ with finite groups: $H_{k l} \equiv T_{m_{k l}}, 1 \leq$ $k, l<\infty$, such that $\mathbb{N}$ can be represented as a disjoint union of $\left\{m_{k l} \mid k, l\right\}$ and some infinite subset. Therefore, $\prod_{i=1}^{\infty} T_{i}$ is represented as the product of $\mathcal{D}=\prod_{k, l \in \mathbb{N}} T_{m_{k l}}$ and the Cantor compactum $\mathcal{C}$. According to Proposition (1.8), there exists a sequence $R_{1} \subset R_{2} \subset \ldots$ of rectangle subsets on $\mathcal{C}$, satisfying (1.8)(7)-(9). Then $\mathcal{D} \times R_{1} \subset$ $\mathcal{D} \times R_{2} \subset \ldots$ is the sequence of the rectangle subsets of $\mathcal{D} \times \mathcal{C}$ also satisfying $(1.8)(7)-(9)$.

By Theorem (3.8), $\left\{r\left(I^{n} \times \mathcal{D} \times R_{i}\right)\right\}$ is an equi-LC ${ }^{n-1}$-family of the Menger compactum $P$. By Remark (2.4), each $\left\{r\left(I^{n} \times \mathcal{D} \times R_{i}\right)\right\}$ is a $Z$-set in $r\left(I^{n} \times \mathcal{D} \times R_{i+1}\right)$. Since $\bigcup_{i=1}^{\infty} r\left(I^{n} \times \mathcal{D} \times R_{i}\right)$ is a dense subset of $P$, it follows by Proposition (1.6) that the pseudo-boundary $\Sigma(P)$ coincides with $\bigcup_{i=1}^{\infty} r\left(I^{n} \times \mathcal{D} \times R_{i}\right)$.

To construct a semi-free action $\Phi$ of $G \subset H$ on $P$ as in Theorem (0.2), we first define a discontinuous action $\Psi$ of $H$ on $Q$. Let $\mathrm{N}\left(B, \mathcal{Q}_{m}\right)$ be the star of a subset $B \subset Q$ with respect to the partition $\mathcal{Q}_{m}=\left\{\mathcal{E}^{\prime} \times \prod_{i=m+1}^{\infty} T_{i} \mid \mathcal{E}^{\prime} \in \mathcal{Q}_{m}^{\prime}\right\}$ of $Q=I^{n} \times \prod_{i=m+1}^{\infty} T_{i}$. Note that $\mathrm{N}\left(B, \mathcal{Q}_{m}\right)$ is a closed neighborhood of $B$ and

(2) $\bigcap_{m=1}^{\infty} \mathrm{N}\left(B, \mathcal{Q}_{m}\right)=r^{-1} r(B)$; and 
(3) if $(x, u, v) \in \operatorname{Bd}\left(\mathrm{N}\left(B, \mathcal{Q}_{m}\right)\right)$, then $\operatorname{ind}(x)=m$.

Let $B_{k}=r^{-1}\left(X_{k}\right) \subset I^{n} \times \mathcal{D} \times \mathcal{C}$. Now the action $\Psi$ of $H$ on $Q$ is defined by setting for $h=\left(h_{k l}\right) \in H$ and $z=(x, u, v) \in I^{n} \times \mathcal{D} \times \mathcal{C}$,

(4) $\Psi(h, z)=\left(x, u^{\prime}, v\right)$, where $u^{\prime}=\left(u_{m_{k l}}^{\prime}\right)$ and

$$
u_{m_{k l}}^{\prime}= \begin{cases}u_{m_{k l}} & \text { if }(x, u, v) \in \operatorname{Int}\left(\mathrm{N}\left(B_{k}, \mathcal{Q}_{m_{k l}}\right)\right), \\ h_{k l} \cdot u_{m_{k l}} & \text { otherwise. }\end{cases}
$$

It is obvious that

(5) $r\left(I^{n} \times \mathcal{D} \times A\right)$ is an invariant subset for every $A \subset \mathcal{C}$.

Let $(x, u, v) \notin B_{k}$ and $g=\left(g_{k l}\right) \in G_{k} \backslash\{e\}$. Since $\mathcal{M}_{k}=\left\{m_{k l} \mid(x, u, v) \in \mathrm{N}\left(B_{k}, \mathcal{Q}_{m_{k l}}\right)\right\}$ is finite, whereas $\mathcal{G}=\left\{l \mid g_{k l} \neq e\right\}$ is infinite, we have

(6) $B_{k}$ is the fixed-point set for every $g=\left(g_{k l}\right) \in G_{k} \backslash\{e\}$.

Next the action $\Phi$ of $H$ on $P$ is defined by $\Phi(h, r(x, u, v))=r(\Psi(h,(x, u, v)))$. As the preimage $r^{-1}(z)$ is a rectangle subset $x \times \mathcal{T}(x, t)$ of $Q$, the action $\Phi$ is welldefined. It follows from (5)-(6) and the formula for $\Phi$ that $\Sigma(P)$ and $\nu(P)$ are invariant subsets and $X_{k}$ is the fixed-point set for every $g \in G_{k} \backslash\{e\}$.

To complete the proof of Theorem (0.2), we need the following fact:

Proposition (4.1). The action $\Phi: G \times P \rightarrow P$ is continuous.

Proof. It suffices to verify that for every $\left(x_{0}, t_{0}\right) \in Q, \epsilon>0$ and $g_{0} \in G$, there exist a neighborhood $O\left(g_{0}\right)$ of $g_{0}$ and $\delta>0$, such that $\operatorname{dist}\left((x, t),\left(x_{0}, t_{0}\right)\right)<\delta$ implies $\alpha_{H}\left(g \cdot(x \times \mathcal{T}(x, t)), g_{0} \cdot\left(x_{0} \times \mathcal{T}\left(x_{0}, t_{0}\right)\right)\right)<\epsilon$, for every $g \in O\left(g_{0}\right)$.

We shall use the upper semi-continuity of the decomposition $\mathcal{T}$ (Proposition (2.1)). Let us choose $\delta>0$ and $p \in \mathbb{N}$, satisfying (8)-(10) in Section 2, and a neighborhood $O\left(g_{0}\right)$ of $g_{0}$ such that the first $p$ coordinates of every element $g \in O\left(g_{0}\right)$ coincide. Then it follows that $g \cdot(x \times \mathcal{T}(x, t))=x \times \mathcal{T}\left(x_{0}, g \cdot t\right)$ and $\alpha_{H}\left(x \times \mathcal{T}(x, g \cdot t), x_{0} \times \mathcal{T}\left(x_{0}, g_{0} \cdot t_{0}\right)\right)<\epsilon$, analogously to the proof of Proposition $(2.1)$.

\section{EPILOGUe}

Let $M$ be a $\mu^{k}$-manifold. By Theorem (1.4), there exist a PL manifold $R$ of dimension $\geq 2 k+1$ with a triangulation $L$, and a proper map $f: R^{(k)} \rightarrow M$ which induces isomorphisms of homotopy groups of $\operatorname{dim}<k$ and of homotopy groups of ends of $\operatorname{dim}<k$, where $R^{(k)}=\left|L^{(k)}\right|, L^{(k)}$ is the $k$-skeleton of $L$. If $R^{(k)}$ would admit a cubical triangulation, then by applying Sections 2-4 word-by-word, we could construct the map $r: R^{(k)} \times \prod T_{i} \rightarrow\left(R^{(k)} \times \prod T_{i}\right) / \mathcal{T}=N^{k}$ into $N^{k}$, which by Theorem (1.3) is a $\mu^{k}$-manifold. The natural projection $\pi^{\prime}: R^{(k)} \times \prod T_{i} \rightarrow R^{(k)}$ generates the proper retraction $\pi: N^{k} \rightarrow R^{(k)}$, which would induce isomorphisms of homotopy groups of $\operatorname{dim}<k$ and of homotopy groups of ends of $\operatorname{dim}<k$. The composition $f \circ \pi$ would then be a proper map between $\mu^{k}$-manifolds which would induce isomorphisms of homotopy groups of $\operatorname{dim}<k$ and of homotopy groups of ends of $\operatorname{dim}<k$. By Theorem (1.5), $f \circ \pi$ would be properly $(k-1)$-homotopic to a homeomorphism and therefore $\left(R^{(k)} \times \prod T_{i}\right) / \mathcal{T}$ would be homeomorphic to $M^{k}$. As in Section 4 , we could construct the desired action of $\prod G_{i}$ on $N=\left(R^{(k)} \times \prod T_{i}\right) / \mathcal{T}$.

Problem (5.1). Is every simplicial complex homeomorphic to a complex which admits a cubical triangulation? 
Without referring to the solvability of this problem, we give an outline of the proof of Theorem (0.2) in the case of $\mu^{k}$-manifold $M$. To this end, we consider the handlebody decomposition $H_{m}=\left\{\operatorname{St}\left(v ; \beta^{m+2} L\right) \mid v \in\left(\beta^{m+1} L\right)^{(0)}\right\}$ of $R^{(k)}$ according to $\beta^{m} L$, where $\beta^{m} L$ is the $m$ th barycentric subdivision of the triangulation $L$ and $\left(\beta^{m+1} L\right)^{(0)}$ are the vertices of the $(m+1)$-st barycentric subdivision.

By $F_{m}$ we denote the union of all the boundaries of all elements of the partition $H_{m}$. Clearly, the analogue of (2) in Section 2 holds:

(1) for every $x \in R^{(k)}$, the set $\left\{m \mid x \in F_{m}\right\}$ has less than $k+1$ elements.

All assertions from Sections 2-4 go through in our case, whereas their proofs do not differ substantially and the reader can supply the details.

Only the fact that the proper retraction $\pi:\left(R^{(k)} \times \prod T_{i}\right) / \mathcal{T} \rightarrow R^{(k)}$ induces isomorphisms of homotopy groups of $\operatorname{dim}<k$ and of homotopy groups of ends of $\operatorname{dim}<k$, requires a proof. Due to the limited space we must leave the details to the readers. However, we wish to point out that they do not significantly differ from the techniques used in our proofs above.

As in Section 4 we can construct the desired action of $\prod G_{i}$ on $\left(R^{(k)} \times \prod T_{i}\right) / \mathcal{T}$, where $\prod T_{i}$ is chosen as $\prod H_{k l} \times \mathcal{C}$.

\section{ACKNOWLEDGEMENTS}

The first author was supported in part by the INTAS grant No. 96-0712. The second author was supported in part by the Ministry of Science and Technology of the Republic of Slovenia grant No. J1-0885-0101-98. We thank the referee for a very careful reading of the manuscript and for contributing several important comments and suggestions.

\section{REFERENCES}

1. S. M. Ageev, An equivariant generalization of Michael's selection theorem, Mat. Zametki 57:4 (1995), 499-508 (in Russian); English transl. in Math. Notes 57:3-4 (1995), 345-350. MR 96h:54017

2. C. Bessaga and A. Pelczyński, Selected Topics in Infinite-dimensional Topology, PWN, Warsaw, 1975. MR 57:17657

3. M. Bestvina, Characterizing k-dimensional universal Menger compacta, Mem. Amer. Math. Soc. 71(380) (1988). MR 89g:54083

4. K. Borsuk, On the imbedding of systems of compacta in simplicial complexes, Fund. Math. 35 (1948), 217-234. MR 10:391b

5. K. Borsuk, Theory of Retracts, PWN, Warsaw, 1967. MR 35:7306

6. G. E. Bredon, Introduction to Compact Transformation Groups, Academic Press, New York, 1972. MR 54:1265

7. A. Chigogidze, K. Kawamura and E. D. Tymchatyn, Nöbeling spaces and pseudo-interiors of Menger compacta, Topol. Appl. 68 (1996), 33-65. MR 97c:54032

8. A. N. Dranishnikov, Free actions of 0-dimensional compact groups, Izv. Akad. Nauk SSSR Ser. Mat. 52:1 (1988), 212-228 (in Russian); English transl. in Math. USSR Izv. 32:1 (1989), 217-232. MR 90e:57065

9. A. N. Dranishnikov, Absolute extensors in dimension $n$ and dimension-raising $n$-soft maps, Uspehi Mat. Nauk 39:5 (1984), 55-95 (in Russian); English transl. in Russian Math. Surv. 39:5 (1984), 63-111. MR 86c:54017

10. V. V. Fedorchuk and V. V. Filippov, General Topology. Basic Constructions, Moscow Univ. Press, Moscow, 1988 (in Russian).

11. Y. Iwamoto, Fixed-point sets of transformation groups of Menger manifolds, their pseudoboundaries and their pseudo-interiors, Topol. Appl. 68 (1996), 267-283. MR 97a:54035]

12. L. S. Pontryagin, Topological Groups, Gordon and Breach, New York, 1966. 
13. K. Sakai, Free actions of zero-dimensional compact groups on Menger manifolds, Proc. Amer. Math. Soc. 122 (1994), 647-648. MR 95c:57057

14. K. Sakai, Semi-free actions of zero-dimensional compact groups on Menger compacta, Proc. Amer. Math. Soc. 125 (1997), 2809-2813. MR 97j:54043

15. E. H. Spanier, Algebraic Topology, Mc Graw-Hill, New York, 1966. MR 35:1007

Department of Mathematics, Brest State University, 224011 Brest, Belarus

E-mail address: ageev@highmath.brsu.brest.by

Institute for Mathematics, Physics and Mechanics, University of Ljubljana, 1001 LuUbluana, Slovenia

E-mail address: dusan.repovs@fmf.uni-lj.si 